\title{
Electrical conductivity of ice VII
}

\author{
Taku Okada' , Toshiaki litaka ${ }^{2}$, Takehiko Yagi ${ }^{3,1}$ \& Katsutoshi Aoki ${ }^{4}$
}

SUBJECT AREAS:

PHASE TRANSITIONS

AND CRITICAL

PHENOMENA

GIANT PLANETS

STRUCTURE OF SOLIDS AND

LIQUIDS

Received

11 April 2014

Accepted

2 July 2014

Published

22 July 2014

Correspondence and requests for materials should be addressed to T.I. (tiitaka@riken.jp) or T.O. (okataku@issp.utokyo.ac.jp)

${ }^{1}$ Institute for Solid State Physics, University of Tokyo, 5-1-5 Kashiwanoha, Kashiwa, Chiba 277-8581, Japan, ${ }^{2}$ Computational Astrophysics Laboratory, RIKEN, 2-1 Hirosawa, Wako, Saitama 351-0198, Japan, ${ }^{3}$ Geodynamics Research Center, Ehime University, 2-5 Bunkyo-cho, Matsuyama, Ehime 790-8577, Japan, ${ }^{4}$ Institute for Materials Research, Tohoku University, 2-1-1 Katahira, Aoba-ku, Sendai, Miyagi 980-8577, Japan.

It was discovered that a peak appears near a pressure of $P_{c}=10 \mathrm{GPa}$ in the electrical conductivity of ice VII as measured through impedance spectroscopy in a diamond anvil cell (DAC) during the process of compression from $2 \mathrm{GPa}$ to $40 \mathrm{GPa}$ at room temperature. The activation energy for the conductivity measured in the cooling/heating process between $278 \mathrm{~K}$ and $303 \mathrm{~K}$ reached a minimum near $P_{c}$. Theoretical modelling and molecular dynamics simulations suggest that the origin of this unique peak is the transition of the major charge carriers from the rotational defects to the ionic defects.

rotons jump among molecules in water and ice. The proton dynamics in water and aqueous solutions has been a major subject of research in physical chemistry for the life sciences. Similarly, the proton dynamics in ice has been a major subject of research in the earth and planetary sciences. Grotthuss proposed the proton relay model for water in $1806^{1}$, and Bjerrum proposed the rotational defect model for ice in $1952^{2}$. There have been innumerable experiments concerning the proton conduction in ice for more than a century ${ }^{3}$, and the corresponding theory has been established through the efforts of multiple researchers ${ }^{4-6}$. In most cases, temperature effects and impurity effects have been addressed, whereas pressure effects ${ }^{7-11}$ have been addressed in only a few cases. Water has a complex phase diagram with many phases (Fig. 1). Under compression at room temperature, liquid water freezes into ice VI at approximately $1 \mathrm{GPa}$ and transforms into ice VII at pressures over $2 \mathrm{GPa}^{12,13}$. Ice VII is a crystal of water molecules formed into a bcc oxygen lattice and a random hydrogen-bond network, which is composed of two interpenetrating but independent ice $I_{c}$ sublattices. As the pressure increases from $2 \mathrm{GPa}$ to $60 \mathrm{GPa}$, the bcc oxygen lattice remains stable with a reduced lattice constant, while the hydrogenbond network evolves from a static random state to a dynamic random state and eventually to a symmetric state. Ice VII exhibits many phenomena originating from its complex proton dynamics, regarding which X-ray diffraction provides very little information. Among these phenomena, superionic ice ${ }^{14}$ is a theoretical phase of water under high temperature and high pressure that is composed of ionised water and has the properties of both a crystal and a liquid: the oxygen ions crystallise in the bcc ( $\mathrm{or} \mathrm{fcc}{ }^{15}$, at extremely high pressures) lattice, whereas the hydrogen ions flow inside the oxygen lattice. It has been suggested that ice-giant planets, such as Uranus and Neptune, may possess a layer of superionic ice ${ }^{16}$. However, experimental evidence of superionic ice has been elusive, partly because of the lack of a clear-cut definition of the phase. Cavazzoni et al. ${ }^{14}$ have identified superionic ice in quantum molecular dynamics simulations by observing an abrupt increase in the proton-diffusion coefficient. Sugimura et al. ${ }^{17}$ have defined it as a state of ice VII with electrical conductivity greater than $10^{-1}(\mathrm{~S} / \mathrm{m})$ because the observed conductivity was found to follow a single Arrhenius-type relation and did not exhibit any discontinuity. Goncharov et al. ${ }^{16}$ have suggested a new phase observed at greater than 47 GPa via Raman spectroscopy to be superionic ice, although no information regarding the proton dynamics was available. Therefore, no phase boundary for superionic ice has yet been confirmed experimentally. Originally, the present study was initiated to investigate the existence of superionic ice by probing proton conduction using the impedance spectroscopy method; however, as reported in this article, an unexpected peak was discovered in the proton conductivity of ice VII measured in the pressure range between $2 \mathrm{GPa}$ and $40 \mathrm{GPa}$ at room temperature. The implications of this discovery are discussed here.

\section{Results}

In the present experiment, high-pressure conditions were generated using a diamond anvil cell (DAC) with flat faces of $0.35 \mathrm{~mm}$ in diameter. Distilled and deionised $\mathrm{H}_{2} \mathrm{O}$ water was loaded into a hole drilled into an electrically insulating gasket composed of cubic boron nitride $(\mathrm{cBN})$ and rhenium $(\mathrm{Re})^{18}$. Two electrodes made of platinum $\mathrm{Pt}$ ) foil with a thickness of $0.002 \mathrm{~mm}$ were placed on the cBN layer (Fig. 2a) in runs \#1-5, whereas gold (Au) electrodes were sputtered onto the culet surfaces of both diamonds (Fig. $2 b$ ) in runs \#6-9. The structure of the 


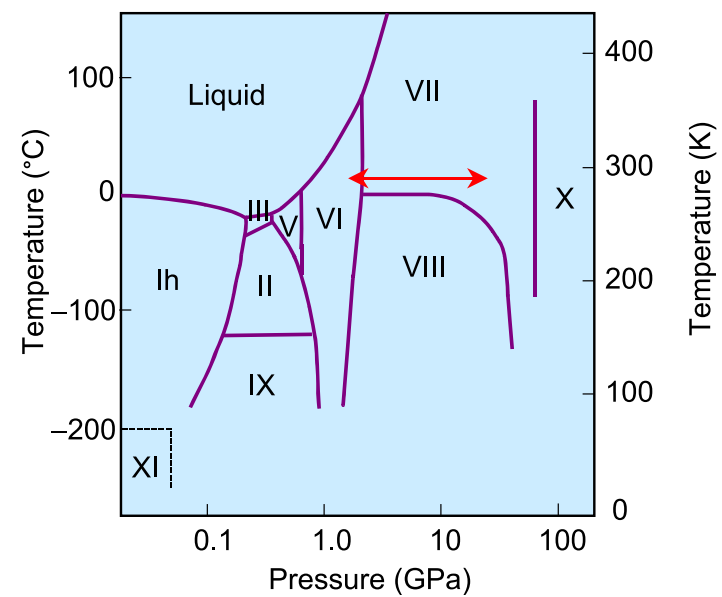

Figure 1 Phase diagram of water with the path of the impedance measurement indicated by a solid red line.

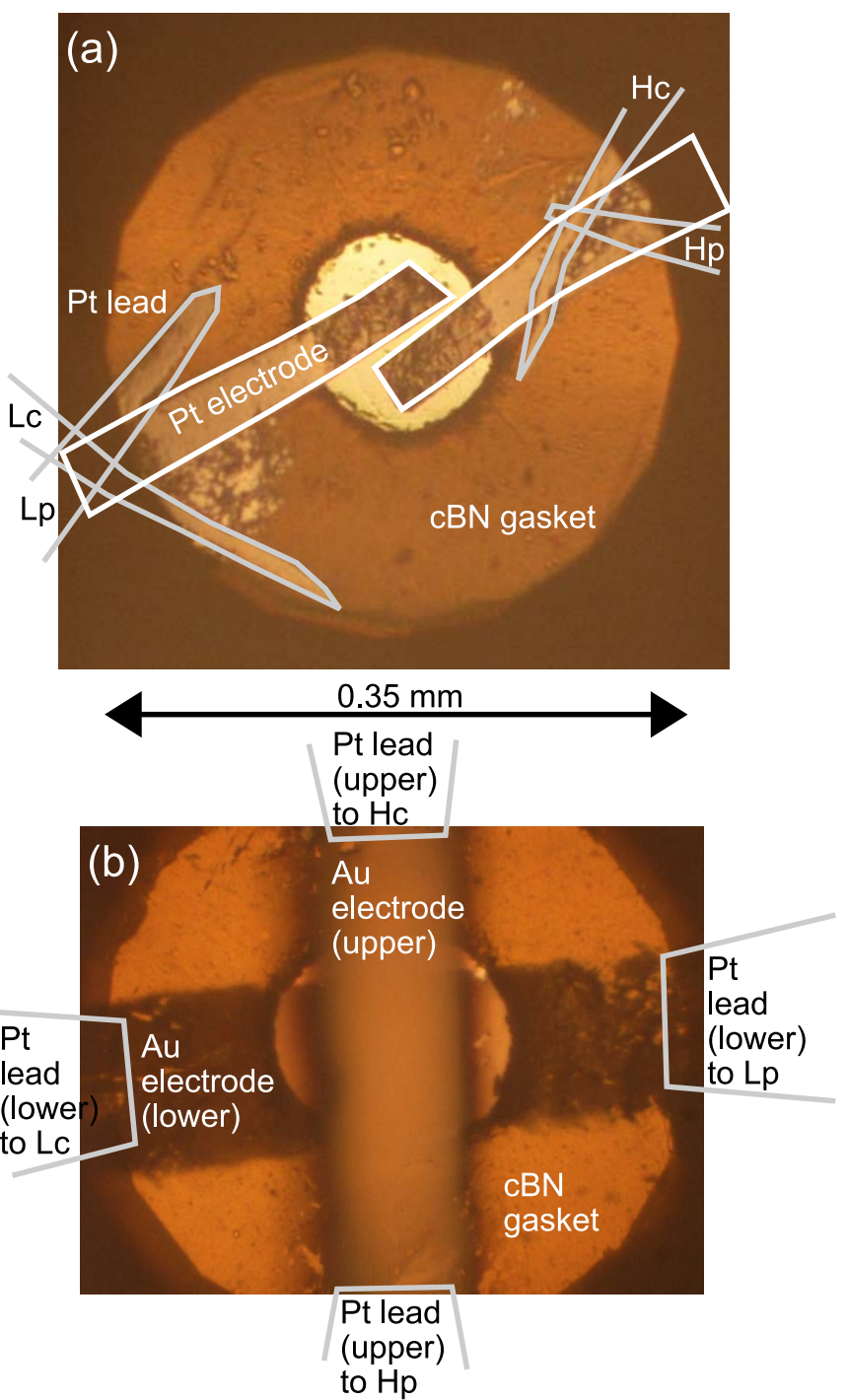

Figure $2 \mid$ Microscopic images of the sample and electrodes in the DAC. (a) Two platinum electrodes placed on the cubic-BN (cBN) layer for runs \#1-5. (b) Gold electrodes sputtered onto the culet surfaces of both diamonds in runs \#6-9, with their ends connected to two platinum leads outside the sample hole for quasi-four-electrode measurements. apparatus is depicted in Supplemental Fig. S1. The width and length of the Pt leads attached to the $\mathrm{cBN}$ layer indicated in the figure were approximately $0.2 \mathrm{~mm}$ and $1.5 \mathrm{~mm}$, respectively. The thickness of the $\mathrm{cBN}$ layer was not measured but was estimated to be $0.05 \mathrm{~mm}$. The pressure was measured by observing the Raman shift of the highfrequency edge of the stressed diamonds ${ }^{19}$. No electrical contact between the two electrodes or between the electrodes and the gasket was detected at any stage in any experiment. The complex impedance of the system $-Z(\omega)=Z^{\prime}(\omega)+i Z^{\prime \prime}(\omega)$, where $Z^{\prime}(\omega)$ and $Z^{\prime \prime}(\omega)$ are the real and imaginary parts, respectively - was measured at room temperature and at pressures from $2.2 \mathrm{GPa}$ to $40 \mathrm{GPa}$; for these measurements, the quasi-four-terminal method was employed at an applied voltage of $1 \mathrm{~V}$ and frequencies, $f$, from $20 \mathrm{~Hz}$ to $1 \mathrm{MHz}$ using an Agilent 4284A precision LCR meter. The obtained impedance data is presented as a Cole-Cole plot (Fig. 3) and was fitted using an equivalent circuit, whose impedance, $Z$, consisted of a sample contribution $\left(\mathrm{R}_{1}, Z_{C P E}\right)$, a parasitic contribution $\left(\mathrm{R}_{2}, \mathrm{C}_{2}\right)$ and an electrode contribution $\left(\mathrm{R}_{0}\right)$ :

$$
Z(\omega)=R_{0}+\left(\frac{1}{R}+\frac{1}{Z_{C P E}(\omega)}\right)^{-1} .
$$

A constant phase element ${ }^{20}, Z_{C P E}(\omega)=\frac{1}{C_{C P E}(i \omega)^{p}}$, is an equivalent electrical circuit component that models an imperfect capacitor with fitting parameters, and it reduces to an ideal capacitor $C$ when $p=1$ and $C_{C P E}=C$. The equivalent capacitance for a $\mathrm{CPE}$ is equal to

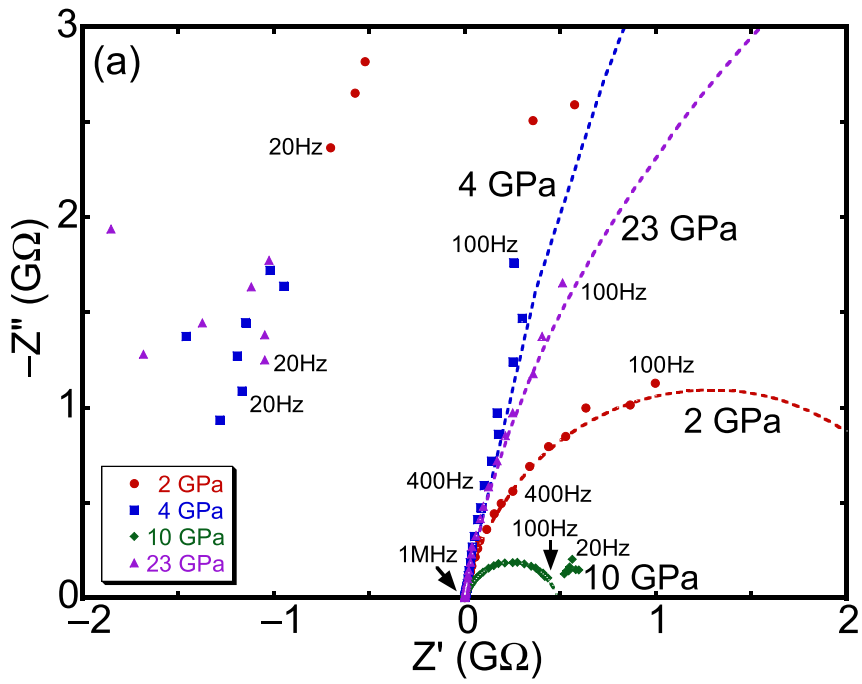

(b)

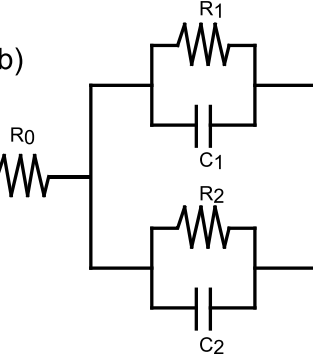

(c)
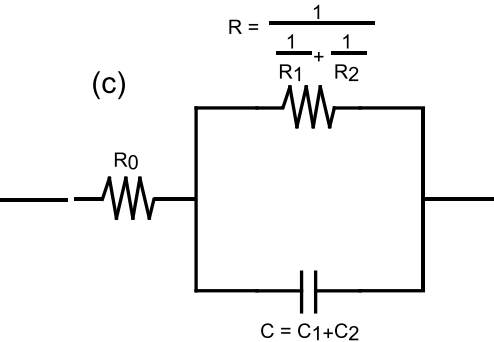

Figure 3 Cole-Cole plot of complex impedance spectra. (a) Spectra for run \#5 showing the real vs. imaginary components of the complex impedance in the frequency range of $1 \mathrm{MHz}$ to $100 \mathrm{or} 20 \mathrm{~Hz}$ at room temperature and various pressures. The dashed lines indicate the spectra of the equivalent circuit. (b) Equivalent circuit consisting of an electrode contribution $\left(\mathrm{R}_{0}\right)$, a sample contribution $\left(\mathrm{R}_{1}, \mathrm{C}_{1}\right)$ and a parasitic contribution $\left(\mathrm{R}_{2}, \mathrm{C}_{2}\right)$. (c) Rearranged equivalent circuit for the equivalent circuit in (b). 


$$
C=C_{C P E}\left(\frac{1}{R C_{C P E}}\right)^{\left(1-\frac{1}{p}\right)}
$$

Data collected at frequencies lower than $\sim 100 \mathrm{~Hz}$ are strongly influenced by the surface effect of the electrodes, as shown in Fig. 3. Note that the diameter of the semi-circle in the Cole-Cole plot indicates the resistance, $\mathrm{R}$, independent of $Z_{C P E}(\omega)$ and $C_{2}$.

Supplementary Fig. S2 presents the pressure dependence of the resistance, $R$, and the capacitance, $C$. There is significant scatter in the data from run to run because of the change in the electrode configuration and, most likely, surface effects. Nevertheless, a common trend can be seen: the resistance exhibits a minimum at $P_{c}$, and the capacitance exhibits both a maximum and a minimum near $P_{c}$.

This trend can be seen more clearly in Supplementary Fig. S3, where the effect of the sample-size variation is reduced by plotting the conductivity, $\sigma=\mathrm{g} / \mathrm{R}$, and permittivity, $\varepsilon=\mathrm{gC}$, using the geometric factor $\mathrm{g}=(d / A)$ for an ideal parallel-plate capacitor with a cross section A and a distance $d$. Because this geometric factor is exact only for $A \gg d^{2}$, a more accurate geometric factor was evaluated using the boundary element method (BEM) ${ }^{21}$ with the electrodes modelled as rectangular plates immersed in uniform dielectrics. The pressure dependence of the result is consistent with the theoretical prediction $^{10,11}$ : the conductivity exhibits a clear peak at $P_{c} \approx 10 \mathrm{GPa}$ (Fig. 4a), and the permittivity, $\varepsilon$, exhibits a minimum near $P_{c}$ (Fig. 4b). The magnitude of the permittivity, however, seems to be overestimated probably because of the contribution from the parasitic capacitance and the difficulty in evaluating the accurate geometric factor taking the apparatus structure into account. The activation energy for the conductivity was evaluated based on the conductivities measured during the processes of cooling and heating between $278 \mathrm{~K}$ and $303 \mathrm{~K}$ at several constant loads. The activation energy reaches a minimum of $0.49 \mathrm{eV}$ near $10 \mathrm{GPa}$ (Fig. 4c).

It is worth noting that the trend in pressure dependence is already recognisable in the resistance and capacitance, as the change in geometry is much smoother than the exponential change in conductivity and permittivity.

Supplementary Fig. S4 presents the cross section, $A$, and the distance, $d$, measured at each pressure and in each run. In runs \#1-5, in which the two platinum electrodes were arranged in skew positions (Fig. 2a), $d$ and $A$ were measured using an optical microscope. In runs \#6-9, in which the two orthogonal gold electrodes were sputtered onto the culet surfaces (Fig. 2b), the cross section, $A$, is defined as the overlapping area of the two electrodes as seen from the pressurising axial direction and was measured at each pressure using an optical microscope. The distance, $d$, is defined as the interval between the culets of the diamond anvils, neglecting the thickness of the sputtered gold electrodes, and was measured using the white-light interference method and the published reflective index of high-pressure ice ${ }^{22}$.

Because the contributions of the sample and those of parasitic effects are indistinguishable in a single run, the impedance of an empty cell with gold electrodes was measured in a separate run (Supplemental Fig. S5), and the parasitic contributions were found to remain nearly constant during the run at $R_{2}=9 \times 10^{9}(\Omega)$ and $C_{2}=2$ $\times 10^{-13}(\mathrm{~F})$. These values are in good agreement with the theoretical estimates, $R_{2}=\rho_{c B N} \frac{d_{c B N}}{L W}=2 \times 10^{10}(\Omega)$ and $C_{2}=\varepsilon_{c B N} \frac{L W}{d_{c B N}}=$ $4 \times 10^{-13}(F)$, which were modelled for a parallel-plate capacitor consisting of a cBN layer (with a thickness of $d_{c B N}=5 \times 10^{-5}(\mathrm{~m})$, a resistivity ${ }^{23}$ of $\rho_{c B N}=10^{8}(\Omega m)$ and a permittivity ${ }^{23}$ of $\left.\varepsilon_{c B N} / \varepsilon_{0}=7.1\right)$ sandwiched between a Re gasket and Pt leads (with a length of $L=1.5$ $\times 10^{-3}(\mathrm{~m})$ and a width of $\left.W=2 \times 10^{-4}(\mathrm{~m})\right)$, as shown in Supplemental Fig. S1. This result implies that the majority of the parasitic contributions originate from this circuit. This knowledge will be useful for the optimisation of the designs of future
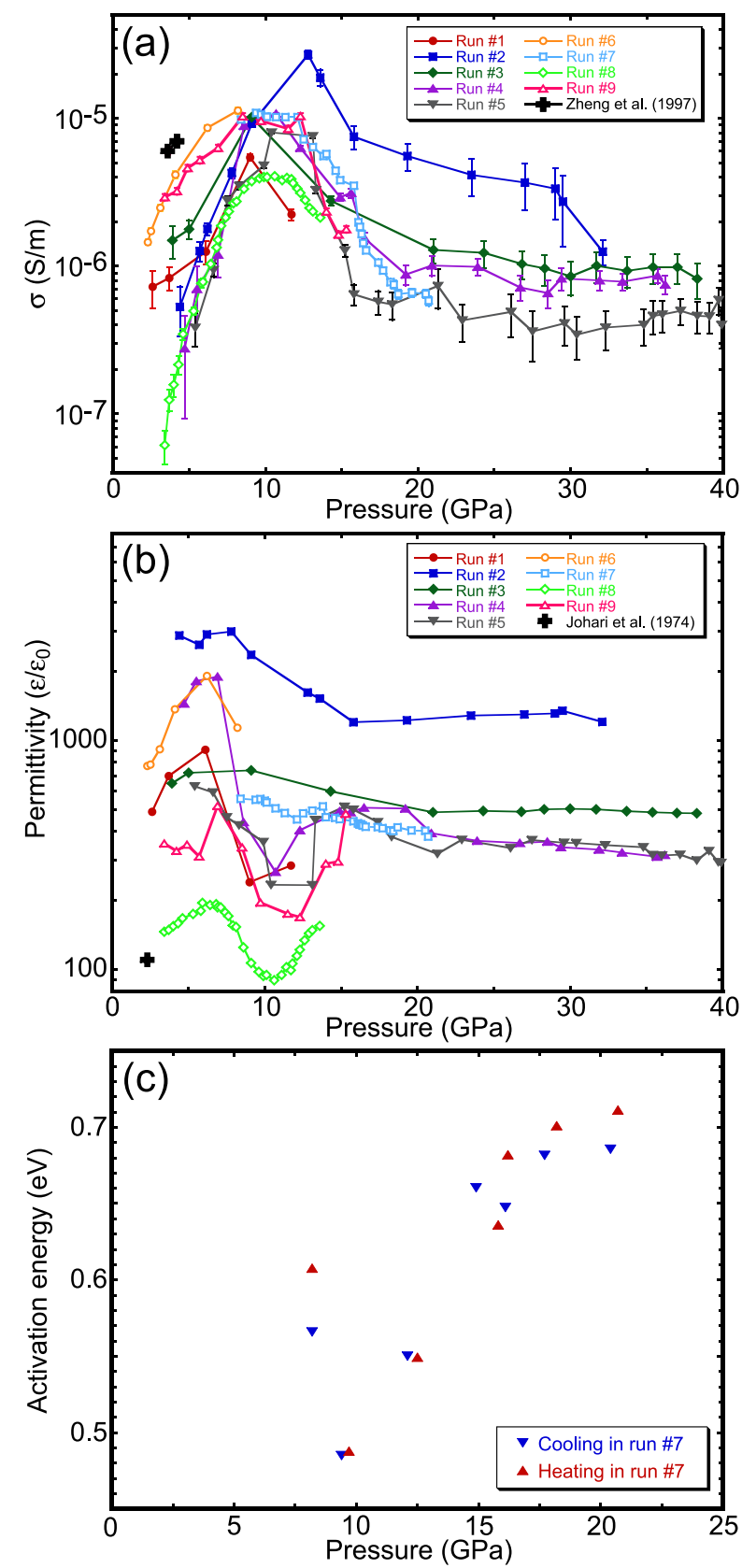

Figure $4 \mid$ Pressure dependence of the physical properties of ice VII. (a) Conductivity calculated from the resistance and dimensions measured for each run and each pressure. (b) Permittivity calculated from the capacitance and dimensions. (c) Activation energy obtained from the Arrhenius plot of the conductivities measured during the processes of cooling and heating between $278 \mathrm{~K}$ and $303 \mathrm{~K}$.

experiments. In the pressure range between $2 \mathrm{GPa}$ and $20 \mathrm{GPa}$, where $R \approx R_{1} \ll R_{2}$, the measured resistance $R$ is approximately equal to the sample contribution, whereas above $20 \mathrm{GPa}$, it is nearly equal to the parasitic contributions. The parasitic contribution of the capacitance is nearly on the order of the sample contribution; which one is larger than the other depends on the experimental conditions of the given run. If $C \approx C_{1} \gg C_{2}$, then the sample contribution is measured, and a maximum and minimum in the capacitance appear near $P_{c}$. If $C \approx C_{2} \gg C_{1}$, then the parasitic contribution is measured, and the capacitance exhibits a monotonic pressure dependence. A similar argument can also be applied to the cells with platinum electrodes (Fig. 2a), although their empty-cell impedance was not measured in this study. 


\section{Discussion}

According to theoretical studies ${ }^{10,11}$, this peak in the conductivity can be attributed to the transition of the major charge carriers in ice VII from the rotational defects to the ionic defects. The theory of electrical conduction in ice ${ }^{5}$ describes such electrical conduction in terms of excitations of proton-defect pairs from the ground state of the hydrogen-bond network ${ }^{4,5}$ that satisfy the ice rules ${ }^{24}$. The conduction mechanism is analogous to that of electrical conduction in an intrinsic semiconductor, which can be described in terms of excitations of electron-hole pairs. The unique feature of electrical conduction in ice is that two types of defect pairs are involved ${ }^{4,5}$, i.e., ionic defect pairs $\left(\mathrm{H}_{3} \mathrm{O}^{+}\right.$and $\left.\mathrm{OH}^{-}\right)$and rotational defect pairs (L-defects and D-defects) $)^{2}$. The former breaks the first ice rule, "two hydrogen atoms exist adjacent to each oxygen atom". The latter breaks the second rule, "exactly one hydrogen atom exists in a bond". Because proton conduction requires both types of defect pairs, the electrical conductivity $(\sigma)$ is determined by the smaller of the ionic $\left(\sigma_{ \pm}\right)$and rotational $\left(\sigma_{D L}\right)$ defect conductivities ${ }^{3,4}$.

$$
\frac{e^{2}}{\sigma}=\frac{e_{ \pm}^{2}}{\sigma_{ \pm}}+\frac{e_{D L}^{2}}{\sigma_{D L}}
$$

where $e$ and $e_{\alpha}$ are the charges of the proton and the defect $\alpha$, respectively. If it is assumed that the density and diffusion constant of the defects follow an Arrhenius-type relation, the defect conductivity can be expressed as

$$
\sigma_{\alpha}=e_{\alpha} n_{\alpha} \mu_{\alpha}=\frac{e_{\alpha}^{2} n_{\alpha}^{0} D_{\alpha}^{0}}{k_{B} T} \exp \left[-\frac{E_{\alpha}^{0}+\gamma_{\alpha} P}{k_{B} T}\right],
$$

where $n_{\alpha}$ and $\mu_{\alpha}$ are the number density and mobility of defect $\alpha$, respectively. The defect charges are $e_{ \pm}=0.62 e$ and $e_{D L}=0.38 e$ for ice $\mathrm{Ih}^{3}$. The constants $n_{\alpha}^{0}$ and $D_{\alpha}^{0}$ are the prefactors of the density and the diffusion constant, respectively; $E_{\alpha}=E_{\alpha}^{0}+\gamma_{\alpha} P$ is the activation energy for the conductance attributable to defect $\alpha$ at a pressure $P$, where $\gamma_{\alpha}$ is the activation volume. In general, $\gamma_{ \pm}<0$ because the barrier between the two possible hydrogen positions in a bond decreases as the pressure increases, whereas $\gamma_{D L}>0$ because a hydrogen bond becomes stronger as the pressure increases. These parameters have been estimated ${ }^{11}$ to be $E_{D L}^{0}=0.503(\mathrm{eV}), E_{ \pm}^{0}=0.596$ $(\mathrm{eV}), \gamma_{D L}=2.59 \times 10^{-2}(\mathrm{eV} / \mathrm{GPa}), \gamma_{ \pm}=-3.64 \times 10^{-2}(\mathrm{eV} / \mathrm{GPa})$, $n_{D L}^{0} D_{D L}^{0}=1.20 \times 10^{24}\left(\mathrm{~s}^{-1} \mathrm{~m}^{-1}\right)$ and $n_{ \pm}^{0} D_{ \pm}^{0}=2.94 \times 10^{19}\left(\mathrm{~s}^{-1} \mathrm{~m}^{-1}\right)$ by fitting experimental results ${ }^{9,25}$ obtained at low pressure. The permittivity $\varepsilon=\varepsilon_{\infty}+\varepsilon_{0} \chi_{s}$ of ice can be represented in terms of the electrical susceptibility,

$$
\chi_{S}=\frac{\left(\frac{\sigma_{ \pm}}{e_{ \pm}}-\frac{\sigma_{D L}}{e_{D L}}\right)^{2}}{\varepsilon_{0} \Phi\left(\frac{\sigma_{ \pm}}{e_{ \pm}^{2}}+\frac{\sigma_{D L}}{e_{D L}^{2}}\right)^{2}},
$$

where $\Phi$ is a constant ${ }^{3}$. A remarkable feature of eq. (3) is that $\chi_{S}$ becomes zero when $\sigma_{ \pm} / e_{ \pm}=\sigma_{ \pm} / e_{D L}$ because the fluxes of the two types of defects are balanced, allowing electrical current to flow without any build-up of polarisation ${ }^{3}$. The features observed in the measured data can be explained using this model. According to eq. (1), the conductivity reaches a maximum at a certain critical pressure, $P_{\mathrm{c}}$ $\approx 10 \mathrm{GPa}$, where $\sigma_{ \pm} / e_{ \pm}^{2}$ and $\sigma_{D L} / e_{D L}^{2}$ become equal. The permittivity has both a maximum and a minimum near $P_{c}$, as seen from eq. (3). The activation energy for $\sigma$ is approximately equal to $E_{ \pm}$below $P_{c}$ and is approximately equal to $E_{D L}$ above $P_{c}$, which explains the minimum at $P_{c}$.

Molecular dynamics (MD) simulations ${ }^{11}$ can provide deeper insight into the microscopic mechanism of this conductivity peak. In the MD simulations, the conductivity was evaluated based on the mean-square deviation (MSD) of the protons:

$$
\left\langle\Delta X^{2}(\tau)\right\rangle=\left\langle(X(t+\tau)-X(t))^{2}\right\rangle,
$$

where $X(t)$ is the position of a proton at time $t$ and a pair of brackets indicates the average over all trajectories. A linear increase in MSD with time indicates diffusion motion with the diffusion constant $D=\frac{1}{6} \frac{d\left\langle\Delta X^{2}(\tau)\right\rangle}{d \tau}$. The calculated MSDs are presented in Supplementary Fig. S6. Each MSD consists of two linear components, implying that two diffusion processes are involved: Below $P_{c}$, protons rotate with the water molecules around the oxygen lattice sites and occasionally jump to neighbouring molecules; this behaviour corresponds to the rapid linear increase in the MSD to the size of a water molecule, followed by a slower linear increase. Above $P_{c}$, protons oscillate in hydrogen bonds and occasionally jump to neighbouring bonds; this behaviour corresponds to the rapid linear increase to the distance between the two stable positions of protons in hydrogen bonds, followed by a slower linear increase. The electrical conductivity was evaluated as $\sigma=\frac{e^{2} n D}{k_{B} T}$, where $n$ is the density of protons, and a pressure dependence similar to that observed experimentally and through thermodynamic modelling was obtained. In all simulations, the MSD of the oxygen atoms remained on the order of atomic thermal vibrations, indicating that the oxygen atoms remain at the lattice sites.

The effects of pressure on the conductivity of ice Ih have been studied by a number of researchers ${ }^{7-9}$. Hubmann ${ }^{8}$ has measured the conductivity of doped ice Ih from ambient pressure to the phase boundary between ice Ih and liquid water $(0.15 \mathrm{GPa})$. The total conductivity could be decomposed into ionic and rotational components, with $\gamma_{ \pm}<0$ and $\gamma_{D L}>0$, respectively. The transition of the charge carriers was not observed at $-25^{\circ} \mathrm{C}$ because the theoretical peak pressure was above the range of stability for ice Ih. The conductivity peak became observable at $-51^{\circ} \mathrm{C}$ and $-61^{\circ} \mathrm{C}$ because the peak pressure decreases as the temperature decreases, thus moving into the stability region for ice Ih. By contrast, the conductivity peak of pure ice VII can be observed even at room temperature by virtue of its much wider pressure range of stability.

The observation of a conductivity peak attributable to a pressureinduced carrier transition in ice VII is reported here for the first time. Previous measurements ${ }^{9,17,26}$ of electrical conductivity (or the diffusion constant) are consistent with the present result; however, these previous measurements were limited to pressure ranges either below or above $P_{c}$. Zheng et al. ${ }^{9}$ have measured the conductivity of ice and water between $0.21 \mathrm{GPa}$ and $4.18 \mathrm{GPa}$ and found that the conductivity decreases from $10^{-5} \mathrm{~S} / \mathrm{m}$ at $1 \mathrm{GPa}$ to $10^{-6} \mathrm{~S} / \mathrm{m}$ at $2 \mathrm{GPa}$ in ice VI and then begins to increase at $2 \mathrm{GPa}$ in ice VII. The protondiffusion constant in ice VII at $400 \mathrm{~K}$ has been measured using infrared reflection spectroscopy by Katoh et al. ${ }^{26}$. The diffusion constant was found to decrease exponentially from $10^{-15}\left(\mathrm{~m}^{2} / \mathrm{s}\right)$ at $10 \mathrm{GPa}$ to $10^{-17}\left(\mathrm{~m}^{2} / \mathrm{s}\right)$ at $63 \mathrm{GPa}$. Sugimura et al. ${ }^{17}$ have measured the conductivity of ice VII at temperatures up to $873 \mathrm{~K}$ and pressures between $20 \mathrm{GPa}$ and $101 \mathrm{GPa}$.

In summary, we discovered an unexpected peak in the electrical conductivity of ice VII as measured through impedance spectroscopy in a diamond anvil cell (DAC) during the process of compression from $2 \mathrm{GPa}$ to $40 \mathrm{GPa}$ at room temperature. This peak can be attributed to the transition of the major charge carriers in ice VII from the rotational defects to the ionic defects.

\section{Methods}

Molecular dynamics simulations. In the molecular dynamics simulations, an empirical water-potential model with all degrees of freedom ${ }^{27,28}$ was employed, instead of the widely used rigid-molecule models ${ }^{29}$, to account for the ionisation of water molecules. The simulation cell for ice VII consisted of 128 water molecules, and the atom trajectories were typically calculated for up to 500 ps at pressures between $2 \mathrm{GPa}$ and $60 \mathrm{GPa}$ and at a temperature of $800 \mathrm{~K}$. The temperature was set higher to account for the quantum zero-point motion of the protons, which effectively lowers 
the diffusion barrier. Therefore, the simulations were not fully quantum, and the results should be taken to be semi-quantitative rather than numerically rigorous. The particle trajectories were computed using the Verlet algorithm ${ }^{30}$, and temperature was controlled by a Nose thermostat ${ }^{31}$. The lattice constant of the cubic simulation cell was fixed to the value calculated via static geometric optimisation within the DFT theory ${ }^{32}$ at a given pressure $P$.

1. de Grotthuss, C. J. T. Sur la décomposition de l'eau et des corps qu'elle tient en dissolution à l'aide de l'électricité galvanique. Ann. Chim. 58, 54-73 (1806).

2. Bjerrum, N. Structure and properties of ice. Science 115, 385-390, doi:10.1126/ science.115.2989.385 (1952).

3. Petrenko, V. F. \& Whitworth, R. W. Physics of ice. (Oxford University Press Inc. 1999).

4. Jaccard, C. Thermodynamics of irreversible processes applied to ice. Phys. Kondens. Mater. 3, 99, doi:10.1007/BF02422356 (1964).

5. Jaccard, C. Étude théorique et expérimentale des propriétés électriques de la glace. Helv. Phys. Acta 32, 89, doi:10.5169/seals-112998 (1959).

6. Hubmann, M. Polarization processes in the ice lattice .1. Approach by thermodynamics of irreversible processes - new experimental-verification by means of a universal relation. Z. Phys. B 32, 127-139 (1979).

7. Chan, R., Davidson, D. \& Whalley, E. Effect of Pressure on the Dielectric Properties of Ice I. J. Chem. Phys. 43, 2376-2383, doi:10.1063/1.1697136 (1965).

8. Hubmann, M. Effects of pressure on the dielectric properties of ice lh single crystals doped with $\mathrm{NH}_{3}$ and HF. J. Glaciology 21, 161-172 (1978).

9. Haifei, Zheng et al. The electrical conductivity of $\mathrm{H}_{2} \mathrm{O}$ at 0.21-4.18 GPa and 20$350^{\circ}$ C. Chinese Sci. Bull. 42, 969-976, doi:10.1007/BF02882610 (1997).

10. Iitaka, T. Proton dynamics in high pressure ice. Low Temp. Sci. 71, 121-124 (2013)

11. Iitaka, T. Simulating proton dynamics in high pressure ices. Rev. High Press. Sci. \& Technol. 23, 124-132 (2013).

12. Bridgman, P. W. The phase diagram of water to $45,000 \mathrm{~kg} / \mathrm{cm}^{2}$. J. Chem. Phys. 5 964-966, doi:10.1063/1.1749971 (1937).

13. Sugimura, E. et al. Compression of $\mathrm{H}_{2} \mathrm{O}$ ice to $126 \mathrm{GPa}$ and implications for hydrogen-bond symmetrization: Synchrotron $\mathrm{x}$-ray diffraction measurements and density-functional calculations. Phys Rev B 77, 214103, doi:10.1103/ PhysRevB.77.214103 (2008).

14. Cavazzoni, C. et al. Superionic and metallic states of water and ammonia at giant planet conditions. Science 283, 44-46, doi:10.1126/science.283.5398.44 (1999).

15. Wilson, H. F., Wong, M. L. \& Militzer, B. Superionic to superionic phase change in water: Consequences for the interiors of Uranus and Neptune. Phys. Rev. Lett. 110, $151102(2013)$

16. Goncharov, A. F. et al. Dynamic ionization of water under extreme conditions Phys. Rev. Lett. 94, doi:10.1103/PhysRevLett.94.125508 (2005).

17. Sugimura, E. et al. Experimental evidence of superionic conduction in $\mathrm{H}_{2} \mathrm{O}$ ice J. Chem. Phys. 137, 194505, doi:10.1063/1.4766816 (2012)

18. Eremets, M. I. et al. Electrical conductivity of xenon at megabar pressures. Phys. Rev. Lett. 85, 2797-2800, doi:10.1103/PhysRevLett.85.2797 (2000).

19. Hanfland, M. \& Syassen, K. A Raman-study of diamond anvils under stress. J. Appl. Phys. 57, 2752-2756, doi:10.1063/1.335417 (1985).

20. Evgenij Barsoukov \& Macdonald, J. R. Impedance Spectroscopy: Theory, Experiment, and Applications. (Wiley-Interscience, 2005).

21. Nishiyama, H. \& Nakamura, M. Form and capacitance of parallel-plate capacitors. IEEE Trans. Components. Packaging and Manufact. Technol. Part A 17, 477-484 (1994).

22. Dewaele, A., Eggert, J. H., Loubeyre, P. \& Le Toullec, R. Measurement of refractive index and equation of state in dense $\mathrm{He}, \mathrm{H}_{2}, \mathrm{H}_{2} \mathrm{O}$, and $\mathrm{Ne}$ under high pressure in a diamond anvil cell. Phys Rev B 67, doi:10.1103/PhysRevB.67.094112 (2003).
23. Spriggs, G. E.: 13.5 Properties of diamond and cubic boron nitride. in SpringerMaterials - The Landolt-Börnstein Database (ed. Beiss, P., Ruthardt, R., Warlimont, H.); doi:10.1007/10858641_7.

24. Bernal, J. \& Fowler, R. A theory of water and ionic solution, with particular reference to hydrogen and hydroxyl ions. J. Chem. Phys. 1, 515-548, doi:10.1063/ 1.1749327 (1933).

25. Whalley, E., Davidson, D. W. \& Heath, J. B. R. Dielectric properties of Ice VII, Ice VIII - a new phase of ice. J. Chem. Phys. 45, 3976, doi:10.1063/1.1727447 (1966).

26. Katoh, E., Yamawaki, H., Fujihisa, H., Sakashita, M. \& Aoki, K. Protonic diffusion in high-pressure ice VII. Science 295, 1264-1266, doi:10.1126/science.1067746 (2002).

27. Kumagai, N., Kawamura, K. \& Yokokawa, T. An interatomic potential model for $\mathrm{H}_{2} \mathrm{O}$ - applications to water and ice polymorphs. Mol. Simul. 12, 177-186, doi:10.1080/08927029408023028 (1994)

28. Kawamura, K. Molecular simulations of mineral-water systems. Chikyukagaku (Geochemistry) 42, 115-132 (2008).

29. Jorgensen, W. \& Madura, J. Temperature and size dependence for Monte-Carlo simulations of TIP4P water. Mol. Phys. 56, 1381-1392, doi:10.1080/ 00268978500103111 (1985)

30. Verlet, L. Computer experiments on classical fluids. I. Thermodynamical properties of Lennard-Jones molecules. Phys Rev 159, 98-103, doi:10.1103/ PhysRev.159.98 (1967).

31. Nose, S. A molecular-dynamics method for simulations in the canonical ensemble. Mol. Phys. 52, 255-268, doi:10.1080/00268978400101201 (1984).

32. Kresse, G. \& Hafner, J. Abinitio molecular-dynamics for liquid-metals. Phys Rev B 47, 558-561, doi:10.1103/PhysRevB.47.558 (1993).

\section{Acknowledgments}

We are grateful to M.I. Eremets and I.A. Trojan for their technical assistance in the impedance spectroscopy measurements during the early stage of this research. This work was supported by KAKENHI (No. 22540489, No. 20103001, No. 20103005 and No. 19310083) from MEXT of Japan and the RIKEN iTHES Project. The MD simulations were performed using the computing facilities of the RICC system at RIKEN.

\section{Author contributions}

T.O. performed the experiment. T.I. performed the theoretical analysis and wrote the manuscript. T.Y. designed the experiment. K.A. was involved in the design of the experiment. All authors discussed the results and commented on the manuscript.

\section{Additional information}

Supplementary information accompanies this paper at http://www.nature.com/ scientificreports

Competing financial interests: The authors declare no competing financial interests. How to cite this article: Okada, T., Iitaka, T., Yagi, T. \& Aoki, K. Electrical conductivity of ice VII. Sci. Rep. 4, 5778; DOI:10.1038/srep05778 (2014).

This work is licensed under a Creative Commons Attribution-NonCommercialNoDerivs 4.0 International License. The images or other third party material in this article are included in the article's Creative Commons license, unless indicated otherwise in the credit line; if the material is not included under the Creative Commons license, users will need to obtain permission from the license holder in order to reproduce the material. To view a copy of this license, visit http:// creativecommons.org/licenses/by-nc-nd/4.0/ 